\title{
EDITORIAL
}

\section{A Nobel endeavour}

\author{
A look back at the microbiologists who have won a Nobel Prize, and at those who have been \\ overlooked, provides a wonderful view of the recent history of microbiology and reveals the \\ central place of microbiology in past and current biological science.
}

The Nobel Prize is the highest honour that a scientist can receive; the very worthy selection of Robert Edwards as recipient of the 2010 prize in Physiology or Medicine for his work on in vitro fertilization is the reward for the completion of a vision that has benefited many over the past 32 years. The list of winners since the first Nobel Prize in Physiology or Medicine was awarded in 1901 contains many microbiologists and indicates the importance of microbiology to biological research. An overview of the Nobel Prizes that have been awarded to microbiologists gives a fascinating view of the progress of microbiology and molecular biology in the twentieth and twenty-first centuries.

Several Nobel Prizes, both early and recent, were awarded for the identification of organisms that cause disease. Robert Koch was honoured in 1905 "for his investigations and discoveries in relation to tuberculosis", which included his now-famous Postulates for the identification of aetiological agents of disease; the discovery that the mosquito is the agent of transmission for malaria and the identification of the malaria parasite were recognized with awards to Ronald Ross, in 1902, and Alphonse Laveran, in 1907, respectively, and Charles Nicolle received his Nobel Prize in 1928 for the finding that epidemic typhus is transmitted by lice. More recently, the identification of Helicobacter pylori and its role in gastritis and peptic ulcer disease netted Barry Marshall and Robin Warren a Nobel Prize in 2005, and in 2008 the Nobel Prize was shared between Harald zur Hausen, for his discovery that human papillomaviruses can cause cervical cancer, and Françoise Barré-Sinoussi and Luc Montagnier, for their discovery of HIV.

The progress of drug discovery can also be revealed by a look at the Nobel winners. Best known is the discovery and development of penicillin by Sir Alexander Fleming, Ernst Chain and Sir Howard Florey, which was recognized in 1945. Gerhard Domagk was awarded the Nobel Prize in 1939 for demonstrating the antibacterial effects of Prontosil, and Selman A. Waksman was the winner in 1952 for his discovery of another antibiotic, streptomycin.

Some microbiologists have won the Nobel Prize for the discovery of basic biological principles. Joshua
Lederberg, who is credited with the discovery of transduction and conjugation, was honoured in 1958 "for his discoveries concerning genetic recombination and the organization of the genetic material of bacteria”. Peyton Rous received his prize in 1966 for his finding that viruses can induce tumour formation, and this prize was shared with George Beadle and Edward Tatum for their description of the 'one gene, one enzyme' hypothesis, another fundamental biological principle. Max Delbrück, Alfred Hershey and Salvador Luria were recognised in 1969 "for their discoveries concerning the replication mechanism and the genetic structure of viruses", and Stanley Prusiner was recognized in 1997 for his discovery of prions.

However, some notable achievements in microbiology have gone unrewarded by the Nobel committee. Some individuals had passed away when the prizes were instated and were thus not eligible; hence, Louis Pasteur and Ignaz Semmelweis never received a Nobel Prize. One eligible but overlooked microbiologist is Martinus Beijerinck. His list of accomplishments includes the discovery of viruses (after a similar description of infectious agents by the Russian scientist Dimitri Ivanovski) and of bacterial nitrogen fixation and sulphate reduction. Similarly, Sergei Winogradski's contributions to the investigation of bacterial metabolism went unrecognized. Their accomplishments may have come too early, as the early Nobel Prizes tended to reward advances that had been made in the previous year. An often cited omission is Oswald Avery, who, together with Colin MacLeod and Maclyn McCarty, identified DNA as the carrier of genetic information. Last, few microbiologists have had as much influence on the current state of the field as Stanley Falkow. His investigations of the pathogenic mechanisms of bacteria and the interaction of bacteria with eukaryotic host cells have provided many important insights into bacterial pathogenesis and laid the groundwork for a vibrant discipline in microbiology. Perhaps his time has yet to come.

This honour roll reveals some of the tremendous progress that has been made in microbiology over the past 110 years, from disease-specific discoveries to fundamental insights. But, as another look back in 100 years' time will reveal, there is still much to be discovered. 